\title{
Polarization-mode interferometry in birefringent single-mode fibers
}

\author{
Luc Thévenaz \\ University of Geneva, Group of Applied Physics, 20 rue Ecole-de-Medecine, 1211 Geneva 4, Switzerland \\ Vincent de Coulon \\ Alphatronix SA, $111 \mathrm{Ch}$. Pont-du-Centenaire, 1228 Geneva, Switzerland \\ Jean-Pierre Von der Weid \\ University of Geneva, Group of Applied Physics, 20 rue Ecole-de-Medecine, 1211 Geneva 4, Switzerland
}

Received February 19, 1987; accepted May 18, 1987

\begin{abstract}
An interferometric technique was used to investigate the relative phase delay between polarization modes in birefringent single-mode fibers. Polarization-mode dispersion is directly deduced from the measurements of relative phase delay at different wavelengths. Relative group delays of $20 \mathrm{fsec}$ can be measured in meter-length samples without the need for light-polarizing devices.
\end{abstract}

In single-mode fibers the polarization-state degeneracy of the fundamental mode is usually removed by either residual or induced anisotropies and internal stresses within the fiber. For most transmission system applications it is desirable to keep the group delay of the two polarization modes as close as possible in order to avoid system-bandwidth limitations. ${ }^{1}$ However, more recent applications require high-birefringence fibers in which the group delays of the polarization modes are made as different as possible. ${ }^{2}$ Typical values of the polarization-mode dispersion (PMD) range from below $1 \mathrm{psec} / \mathrm{km}$ in normal transmission systems to above $1 \mathrm{nsec} / \mathrm{km}$ in polarization-maintaining fibers.

Frequency-domain techniques have been used for birefringence measurements of long $(\sim 1-\mathrm{km})$ singlemode fibers with reported resolution of $3 \mathrm{psec} / \mathrm{km} .{ }^{3}$ Interferometric techniques have improved this time resolution down to about 0.1 psec in both long ${ }^{4}$ and short ${ }^{5}$ samples. All these methods involve careful alignment of launching optics and linear polarizers and the search for birefringence axes, so that a complete measurement requires great care and time. In a recent paper we reported on interferometric measurements of PMD in short samples $(\sim 2 \mathrm{~m})$ of high-birefringence fibers (PMD $\geq 0.4 \mathrm{psec} / \mathrm{m}$ ) without using linear polarizers and parallel-beam optics. ${ }^{6}$ In the present work the interferometric method has been used to investigate the phase relationship between the polarization modes and to measure the PMD and the phase delay of birefringent single-mode fibers.

When nearly monochromatic light of angular frequency $\omega$ is launched into a Michelson interferometer with dispersive but nonbirefringent legs, the intensity of the detected interference signal can be written as the real part of ${ }^{7}$

$$
\begin{aligned}
f\left(l_{0}\right)= & \exp \left[i\left(\beta l-\beta_{0} l_{0}\right)\right] \int \mathrm{d} \omega^{\prime}\left|F\left(\omega^{\prime}-\omega\right)\right|^{2} \\
& \times \exp \left[i\left(\tau l-\tau_{0} l_{0}\right)\left(\omega^{\prime}-\omega\right)\right]
\end{aligned}
$$

where $\beta l$ and $\beta_{0} l_{0}$ are the phase delays in the sample and reference arms, respectively, $\tau l=(\mathrm{d} \beta / \mathrm{d} \omega) / l$ and $\tau_{0} l_{0}=\left(\mathrm{d} \beta_{0} / \mathrm{d} \omega\right) / l_{0}$ are the corresponding group delays, and $\left|F\left(\omega^{\prime}-\omega\right)\right|^{2}$ is the power spectrum of the light source centered at the frequency $\omega$.

In our setup, the length $l_{0}$ of the reference arm can be accurately determined and varied by elastically elongating a single-mode fiber with a computer-controlled $0.1-\mu \mathrm{m}$ stepping-motor translation stage. ${ }^{7}$ When the length $l_{0}$ of the reference leg is swept at a constant rate, the defiling interference fringes give rise to a detected ac signal whose amplitude, which is measured directly by a tuned amplifier, ${ }^{7}$ is the Fourier transform of the power spectrum of the light source centered at the position for which the two legs have equal group delays. The light source is a halogen lamp filtered by a monochromator so that its power spectrum is approximately a square-shaped function. Therefore its Fourier transform can be well described by $\operatorname{sinc}\left[\Omega\left(\tau l-\tau_{0} l_{0}\right)\right]$, where $\Omega$ is a constant, depending on the monochromator wavelength and spectral width.

When the sample fiber is birefringent, the polarization modes are no longer degenerate and have their own phase and group velocity. Because different polarizations are orthogonal, light having traveled in one arm can only interfere with light of the same initial polarization mode from the other arm. When unpolarized light is launched into the interferometer with one birefringent arm, the polarization modes are equally populated with statistically independent pho- 


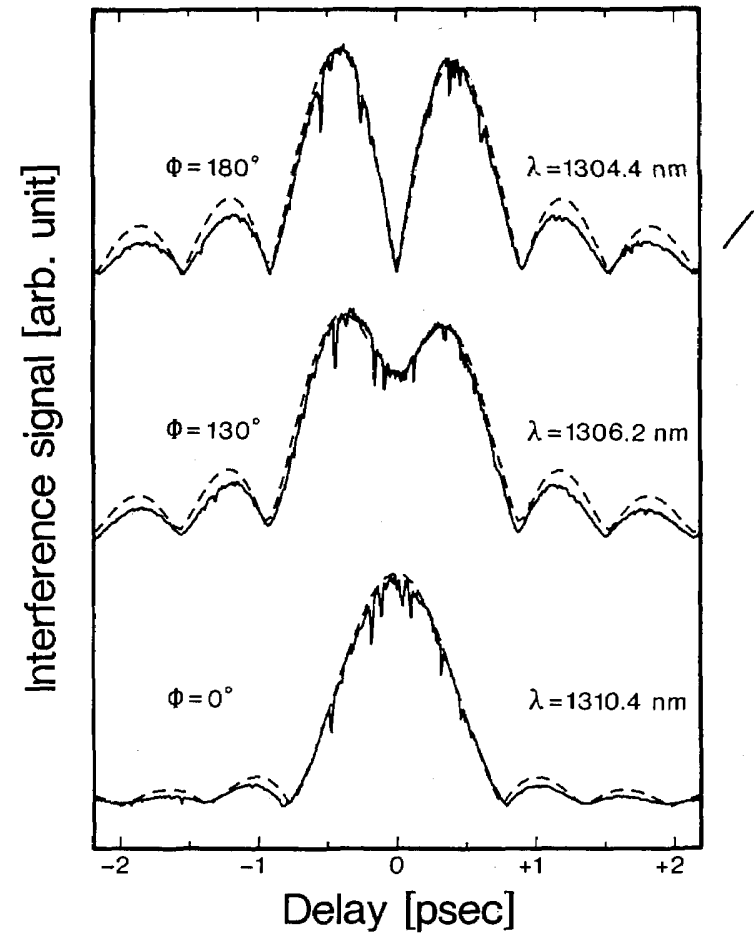

Fig. 1. Amplitude of interference signal for different phase delays between polarization modes. Dashed lines are the best functions fitting the experimental curves following Eq. (3).

tons so that the detected signal is the sum of two independent interference signals, as described by Eq. (1), each corresponding to one polarization mode

$$
\begin{aligned}
f_{B}\left(l_{0}\right)= & A_{1}\left(l_{0}\right) \exp \left[i\left(\beta_{1} l-\beta_{0} l_{0}\right)\right]+A_{2}\left(l_{0}\right) \\
& \times \exp \left[i\left(\beta_{2} l-\beta_{0} l_{0}\right)\right],
\end{aligned}
$$

where $A_{i}\left(l_{0}\right)=a_{i} \operatorname{sinc}\left[\Omega\left(\tau_{i} l-\tau_{0} l_{0}\right)\right]$ is the amplitude of the interference signal from the mode $i$. by

The amplitude of the resulting signal is then given

$$
\begin{aligned}
A\left(l_{0}\right)= & \left\{A_{1}^{2}\left(l_{0}\right)+A_{2}{ }^{2}\left(l_{0}\right)\right. \\
& \left.+2 A_{1}\left(l_{0}\right) A_{2}\left(l_{0}\right) \cos \left[\left(\beta_{1}-\beta_{2}\right) l\right]\right\}^{1 / 2} .
\end{aligned}
$$

It can therefore be seen that the total interference signal contains information about the relative phase delay $\left(\beta_{1}-\beta_{2}\right) l$, the group-delay difference $\left(\tau_{1}-\tau_{2}\right) l$ between polarization modes being implicitly contained in $A_{1}\left(l_{0}\right)$ and $A_{2}\left(l_{0}\right)$.

When the product of fiber birefringence by its length is much greater than the coherence length of the light source, the phase-delay information is lost, and the group-delay difference can be directly measured as the distance between the centers of the two functions $A_{1}\left(l_{0}\right)$ and $A_{2}\left(l_{0}\right){ }^{6}$

When the two patterns overlap the phase delay must be considered, and, since it depends on wavelength, it is possible to change from in-phase to out-ofphase interferograms by properly changing the wavelength of the light source. These situations are clearly illustrated in Fig. 1, where one can see the effect of the phase delay between both the polarization modes over a full $\pi$-phase range between 1304.4 and $1310.4 \mathrm{~nm}$, with a 7-nm spectral width in a 20.5-cm-long Fujikura PANDA fiber. The interferometric method can therefore be extended to lower birefringence levels by properly considering the phase relationship between the two polarization modes. Furthermore, the groupdelay difference can also be obtained from the slope of the phase delay versus wave-number curve ${ }^{8}$

$$
\frac{\mathrm{d} \Phi}{\mathrm{d} k}=c\left(\tau_{1}-\tau_{2}\right) l,
$$

where $k$ is the vacuum wave number of the incident light.

The phase delay as a function of the wave number in the 1.3- $\mu \mathrm{m}$ spectral region is plotted in Fig. 2 for the same 20.5-cm Fujikura PANDA fiber. The corresponding PMD calculated from the slope of the $\Phi(k)$ curve is in excellent agreement with the measurement in a longer fiber sample. ${ }^{6}$

The agreement between the PMD measured by polarization-mode interferometry in short samples and the direct measurement of the group-delay difference in long samples strongly supports polarization-mode interferometry as an accurate method for measuring PMD in fibers for which the polarization-mode interference patterns cannot be well enough resolved for samples of reasonable length to be used in an interferometer. We performed phase-delay versus wavelength measurements on several 1.85-m-long fibers, with induced birefringence resulting from either core ellipticity or residual stresses in the circular core. Results for typical samples are shown in Table 1 together with significant parameters.

The expected PMD can be estimated theoretically in the case of elliptical core fibers. Taking into account both stress-induced and geometrical ${ }^{2}$ sources of birefringence, and using the stress optical coefficient of fused silica, the expected PMD of fiber A is $~ 100$ $\mathrm{fsec} / \mathrm{m}$, in quite good agreement with the experimental results. In the case of fibers with circular cores such as fibers $\mathrm{C}$ and $\mathrm{D}$, no theoretical prediction can be made because the internal stresses are unknown.

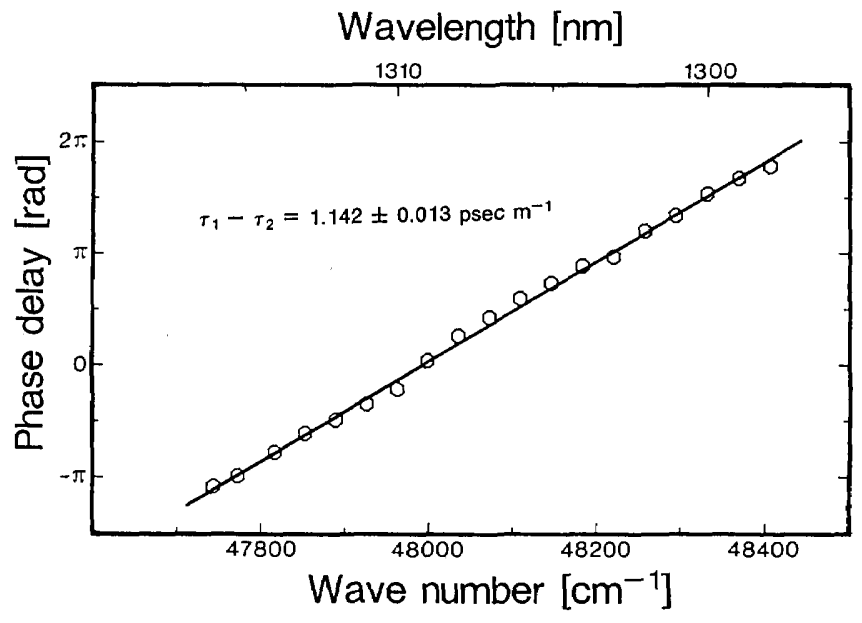

Fig. 2. Phase delay versus wave number and fitted PMD. 
Table 1. Characteristics and Polarization-Mode Dispersion Measurements of Fibers with Geometrical or Stress-Induced Anisotropies

\begin{tabular}{|c|c|c|c|c|}
\hline \multirow[b]{2}{*}{ Characteristics } & \multicolumn{4}{|c|}{ Fibers } \\
\hline & $\mathrm{A}$ & $\mathrm{B}$ & $\mathrm{C}$ & $\mathrm{D}$ \\
\hline $\begin{array}{l}\text { Mean core diameter } \\
(\mu \mathrm{m})\end{array}$ & 6.7 & 9.45 & 6.9 & 5.1 \\
\hline Ellipticity & 0.21 & 0.09 & 0 & 0 \\
\hline $\begin{array}{l}\Delta n^{+}\left(10^{-3}\right) \\
\quad(\text { core })\end{array}$ & 12 & 6.5 & 4.5 & 13 \\
\hline $\begin{array}{l}\Delta n^{-}\left(10^{-3}\right) \\
\quad \text { (inner cladding) }\end{array}$ & 0 & 0 & -2.0 & -2.2 \\
\hline PMD (fsec/m) & 84 & 24 & 23 & 25 \\
\hline
\end{tabular}

Nevertheless, the method can be used to qualify the level of internal stresses within the fiber. We observed that depressed inner-cladding fibers with circular geometry are systematically more birefringent than matched cladding fibers with the same geometry. This means that the smaller expansion coefficient of the inner-cladding material induced by fluorine doping $^{2}$ gives rise to internal stresses and PMD within the fiber.

It should be noted however that the extrapolation of PMD data obtained on a short fiber sample to large lengths of fiber must take into account some degree of mode coupling that depends on both the fiber birefringence and the external perturbations. Assuming a total random coupling between polarization modes, a $l^{1 / 2}$ dependence is expected. ${ }^{1}$ Fiber $\mathrm{C}$ in this case would have a PMD of $\sim 0.6 \mathrm{psec} / \mathrm{km}$ instead of the 23 $\mathrm{psec} / \mathrm{km}$ expected from the linear dependence with mode coupling neglected.

The limitations of this method for measuring the birefringence are related to the fringe-pattern overlap of the two polarization modes that is given by the product of the fiber birefringence and its length and by the spectral width of the light source. By using a 3$\mathrm{nm}$ spectral width and a $1.85-\mathrm{m}$-long fiber sample, a PMD of $0.5 \mathrm{psec} / \mathrm{m}$ can be measured by this technique with $\sim 1 \%$ accuracy. Higher birefringences are better measured with a larger spectral width by direct subtraction of the group delays in the nonoverlapping regime. ${ }^{6}$ The best time resolution of the method for low birefringence is limited by the accuracy of the theoretical fit for close fringe patterns. With a 9-nm spectral width this limit is estimated to be $\sim 20 \mathrm{fsec}$, mainly because of the low intensity of the signal in counterphase. The smaller PMD that can be measured by this method is therefore about $10 \mathrm{fsec} / \mathrm{m}$ in a 2-m sample.

The most important contribution of the polarization-mode interferometry is the access to the relative phase delay between the polarization modes in a birefringent fiber without the need for linear polarizers and parallel beam optics. The method can be directly used to determine the birefringence and internal stresses of standard telecommunication fibers as well as highly birefringent fibers. In addition, the all-fiber Michelson interferometer leaves the mirrored end of the test sample free for the application of mechanical perturbations such as lateral or longitudinal stress, bending, or torsion. The present technique is therefore a simple and powerful tool for analyzing the behavior of phase and group delay of birefringent fibers under external perturbations.

Jean-Pierre Von der Weid is on leave from the Department of Physics, PUC/RJ, CP38075, Rio de Janeiro, Brazil.

\section{References}

1. I. P. Kaminov, IEEE J. Quantum Electron. QE-17, 15 (1984).

2. S. C. Rashleigh, IEEE J. Lightwave Technol. LT-1, 312 (1983).

3. M. Monerie and P. Lamouler, Electron. Lett. 18, 998 (1982).

4. K. Mochizuki, Y. Namihira, and H. Wakabayashi, Electron. Lett. 17, 153 (1981).

5. N. Shibata, M. Tsubokawa, and S. Seikai, Opt. Lett. 10, 92 (1985).

6. J. P. Von der Weid, L. Thévenaz, and J. P. Pellaux, Electron. Lett. 23, 151 (1987).

7. L. Thévenaz, J. P. Pellaux, and J. P. Von der Weid, IEEE J. Lightwave Technol. (in press).

8. S. C. Rashleigh, Opt, Lett. 8, 336 (1983). 